\title{
ALGORITHM FOR CREATING TECHNICAL SYSTEMS FOR REGULATING THE HYDROTHERMAL REGIME OF AGROPHYTOCENOSIS USING THE METHOD OF MORPHOLOGICAL COMBINATOR
}

\author{
V. V. Borodychev, M. N. Lytov \\ Federal State Budget Science Center "All-Russian Scientific Research Institute of Hydrotechnics \\ and Land Reclamation named after A.N. Kostyakov»
}

\section{Summary}

An algorithm for creating technical systems for regulating the hydrothermal regime of agrophytocenosis based on the method of morphological combinatorics has been developed. The algorithm is constructed in such a way that a technical solution with a higher priority always replaces a technical solution with a lower priority, which allows finding the best design for all possible options for the implementation of design features for a set of functional elements of the system.

Abstract
Introduction. The relevance of research is determined by the practical potential of new irrigation and drainage technologies that provide the ability to comprehensively regulate the hydrothermal regime of agrophytocenosis and protect crops from climatic risks. These are quite serious advantages, almost defining a new stage in the implementation of irrigation and drainage technologies in open ground conditions. Today, there is an increasing need for a systematic approach to the implementation of the creative process to create new, highly efficient structures, new technical solutions that expand the functionality of irrigation and drainage systems in terms of regulating the hydrothermal regime of agrophytocenosis. The purpose of this study is to develop an algorithm for creating new designs of irrigation and drainage systems to regulate the hydrothermal regime of agrophytocenosis using the method of morphological combinatorics. Object. The object of research is the design of irrigation and drainage systems with extended functionality for regulating the hydrothermal regime of agrophytocenosis. Materials and methods. The working hypothesis of the study is the assumption about the possibility of automating the process of creating highly efficient designs of irrigation and drainage systems based on the method of morphological combinatorics. The method is sufficiently formalized, which makes it possible, on its basis, to develop computer algorithms for solving specific technical problems. The method of morphological combinatorics involves the selection of sets of constructive or any other features of a technical system, and for each of the features, alternative options are selected. To solve the problem in an automatic mode, it is important that it is possible to organize an enumeration of the options for the execution of these signs. That is, it is necessary that the options for the implementation of a constructive feature be characterized by some quantitative step, or that these options be organized into lists that would be available for enumeration. Results and conclusions. The basis for the creation of irrigation and drainage systems that provide comprehensive regulation of the hydrothermal regime of agrophytocenosis is based on the principle of combining various methods of irrigation. Such systems are complex in design and impose special requirements on the operation of all functional elements; they have fundamentally new functional blocks in comparison with the known designs of irrigation and drainage systems. As in any complex technical system, the created technical solutions have many alternative implementations, and changes in even the most imperceptible structural module of a functional element can significantly affect the operation of the entire system as a whole. The use of the method of morphological combinatorics allows, to a certain extent, to automate the process of engineering creativity, organizing the search and comparison of all combinations with various realizations of the design features of the functional elements of the technical system. Research, based on the method of morphological combinatorics, has developed an algorithm for creating technical systems for regulating the hydrothermal regime of agrophytocenosis. The algorithm includes a system for identifying options for the implementation of a technical system for regulating the hydrothermal regime of agrophytocenosis, functional modules for enumerating combinations of possible design implementations, assessing the consistency of design solutions in various combinations of pos- 
sible implementations, a module for optimizing design parameters when introducing changes for the evaluated implementation option, a module for assessing compliance with the requirements to such technical systems, modules for evaluating the priority criterion of a technical solution, as well as comparing a new and previous technical solution according to this criterion. The algorithm is constructed in such a way that a technical solution with a higher priority always replaces a technical solution with a lower priority. This allows you to find the best technical solution for all possible options for the implementation of design features for a set of functional elements of the system. The algorithm allows automating the process of finding the best design solutions when creating irrigation and drainage systems for the integrated regulation of the hydrothermal regime of agrophytocenosis.

Key words: agrophytocenosis, hydrothermal regime, irrigation and drainage system, creation of structures, morphological combinatorics, algorithm.

Citation. Borodychev V.V., Lytov M.N. Algorithm for creating technical systems for regulating the hydrothermal regime of agrophytocenosis using the method of morphological combinator. Proc. of the Lower Volga Agro-University Comp. 2021. 2(62). 314-330 (in Russian). DOI: 10.32786/2071-94852021-02-33.

Author's contribution. All authors of this research paper have directly participated in the planning, execution, or analysis of this study. All authors of this paper have read and approved the final version submitted.

Conflict of interest. The authors declare no conflict of interest.

\title{
УДК 631.67:581.55
}

\section{АЛГОРИТМ СОЗДАНИЯ ТЕХНИЧЕСКИХ СИСТЕМ ДЛЯ РЕГУЛИРОВАНИЯ ГИДРОТЕРМИЧЕСКОГО РЕЖИМА АГРОФИТОЦЕНОЗА С ИСПОЛЬЗОВАНИЕМ МЕТОДА МОРФОЛОГИЧЕСКОЙ КОМБИНАТОРИКИ}

\author{
В. В. Бородычев, доктор сельскохозяйственных наук, академик РАН \\ М. Н. Лытов, кандидат сельскохозяйственных наук, доцент \\ ФГБНУ Всероссийский научно-исследовательский \\ институт гидротехники и мелиорации им. А. Н. Костякова (Волгоградский филиал)
}

Дата поступления в редакцию 15.03.2021

Дата принятия к печати 28.05.2021

Актуальность. Актуальность исследований определяется практическим потенциалом новых гидромелиоративных технологий, обеспечивающих возможность комплексного регулирования гидротермического режима агрофитоценоза и защиты посевов от климатических рисков. Это достаточно серьёзные преимущества, без малого определяющие новый этап реализации гидромелиоративных технологий в условиях открытого грунта. Сегодня все большее ощущается потребность в системном подходе к реализации творческого процесса по созданию новых, высокоэффективных конструкций, новых технических решений, расширяющих функционал гидромелиоративных системы в плане регулирования гидротермического режима агрофитоценоза. Целью настоящего исследования является разработка алгоритма создания новых конструкций гидромелиоративных систем для регулирования гидротермического режима агрофитоценоза с использованием метода морфологической комбинаторики. Объект. Объектом исследований являются конструкции гидромелиоративных системы с расширенным функционалом по регулированию гидротермического режима агрофитоценоза. Материалы и методы. Рабочей гипотезой исследования является предположение о возможности автоматизации процесса создания высокоэффективных конструкций гидромелиоративных систем на основе метода морфологической комбинаторики. Метод в достаточной мере формализуем, что позволяет на его основе разрабатывать компьютерные алгоритмы для решения конкретных технических задач. Метод морфологической комбинаторики предполагает выделение совокупностей конструктивных или каких-либо других признаков технической системы, причем для каждого из признаков подбираются альтернативные варианты. Для решения задачи в автоматическом режиме важно, чтобы можно было организовать перебор вариантов исполнения этих признаков. 
То есть необходимо, чтобы варианты реализации конструктивного признака характеризовались каким-либо количественным шагом, либо чтобы эти варианты были организованы в списки, которые были бы доступны перебору. Результаты и выводы. В основу создания гидромелиоративных систем, обеспечивающих комплексное регулирование гидротермического режима агрофитоценоза, положен принцип комбинирования различных способов орошения. Такие системы сложны в конструктивном исполнении и предъявляют особые требования к работе всех функциональных элементов, имеют принципиально новые функциональные блоки в сравнении с известными конструкциями гидромелиоративных систем. Как и в любой сложной технической системе, создаваемые технические решения имеют множество альтернативных вариантов реализаций, причем изменения даже в самом незаметном конструктивном модуле функционального элемента могут существенно отражаться на работе всей системы в целом. Использование метода морфологической комбинаторики позволяет в определенной мере автоматизировать процесс инженерного творчества, организуя перебор и сравнение всех комбинаций с различными реализациями конструктивных признаков функциональных элементов технической системы. Исследованиями на основе метода морфологической комбинаторики разработан алгоритм создания технических систем для регулирования гидротермического режима агрофитоценоза. Алгоритм включает систему идентификации вариантов реализации технической системы для регулирования гидротермического режима агрофитоценоза, функциональные модули перебора комбинаций возможных конструктивных реализаций, оценки согласованности конструктивных решений в различных комбинациях возможных реализаций, модуля оптимизации конструктивных параметров при введении изменений по оцениваемому варианту реализации, модуля оценки соответствия требованиям, предъявляемым к таким техническим системам, модулей оценки критерия приоритета технического решения, а также сравнения нового и предшествующего технического решения по этому критерию. Алгоритм построен так, что техническое решение, отличающееся большим приоритетом, всегда замещает техническое решение с меньшим приоритетом. Это позволяет найти наилучшее техническое решение при всех возможных вариантах реализации конструктивных признаков для совокупности функциональных элементов системы. Алгоритм позволяет автоматизировать процесс поиска наилучших конструктивных решений при создании гидромелиоративных систем для комплексного регулирования гидротермического режима агрофитоценоза.

Ключевые слова: агрофитоценозы, гидротермический режим агрофиточенозов, морфологическая комбинаторика, гидротехнические мелиоращии.

Цитирование. Бородычев В. В., Лытов М. Н. Алгоритм создания технических систем для регулирования гидротермического режима агрофитоценоза с использованием метода морфологической комбинаторики. Известия НВ АУК. 2021. 2(62). 314-330. DOI: 10.32786/2071-9485-2021-02-33.

Авторский вклад. Все авторы настоящего исследования принимали непосредственное участие в планировании, выполнении или анализе данного исследования. Все авторы настоящей статьи ознакомились и одобрили представленный окончательный вариант.

Конфликт интересов. Авторы заявляют об отсутствии конфликта интересов.

Введение. Гидротехнические мелиорации по определению направлены на улучшение неблагоприятного водного режима почвы, оказывают непосредственное влияние на геологические круговороты воды в природе. В климатической зоне избыточного увлажнения осуществляются мероприятия по отведению влаги из переувлажненной почвы, а в засушливых климатических зонах дефицит природного увлажнения компенсируется оросительными мелиорациями. Однако уже давно ясно, что изменение условий увлажнения оказывает влияние не только на формирование водного режима мелиорированных земель, но и на целый комплекс сопутствующих факторов. Одним из таких факторов является температурный режим мелиорируемых земель и формируемых на этих землях агрофитоценозов $[6,7,11,12,13]$. Формирование водного режима земель как процесс, динамический и включающий фазовые переходы воды, непосред- 
ственно связано с изменением баланса тепла на мелиорируемых территориях. Этот ресурс уже неоднократно предлагалось использовать, были построены модели водо- и теплообмена в системе почва - растение - атмосфера, разработаны первые способы регулирования микроклимата, базирующиеся на гидромелиоративных технологиях [4, 10]. При практическом освоении приемов гидромелиоративного регулирования микроклимата в посевах стала понятна необходимость учета перекрёстного регулирования факторов жизни, когда одно мелиоративное действие имеет сразу несколько точек приложения. Сегодня видится целесообразным не просто учет проблемы перекрестного регулирования факторов жизни при осуществлении мелиоративных мероприятий, но управление этим процессом комплексно на базе единой технической системы. Такой подход может быть реализован на основе гидромелиоративных систем с возможностью одновременного или поочередного проведения поливов различными способами $[5,9]$. Концепт технической гидромелиоративной системы с возможностью проведения поливов различными способами предполагает возможность регулирования гидротермического режима агрофитоценоза и комплексную протекцию посевов от климатических рисков [2]. Это достаточно серьёзные преимущества, без малого определяющие новый этап реализации гидромелиоративных технологий в условиях открытого грунта. В то же время остаются нерешенными важные вопросы, проблемные как с точки зрения практического освоения новых гидромелиоративных технологий, так и в собственно теории таких систем. Необходим системный подход в реализации творческого процесса по созданию новых, высокоэффективных конструкций, новых технических решений, обеспечивающих возможность комплексного использования гидромелиоративных технологий для регулирования гидротермического режима агрофитозенозов и защиты посевов от климатических рисков. Целью настоящего исследования является разработка алгоритма создания новых, высокоэффективных конструкций гидромелиоративных систем для регулирования гидротермического режима агрофитоценоза с использованием метода морфологической комбинаторики.

Материалы и методы. Рабочей гипотезой исследования является предположение о возможности автоматизации процесса создания высокоэффективных конструкций гидромелиоративных систем на основе метода морфологической комбинаторики. Метод в достаточной мере формализуем, что позволяет на его основе разрабатывать компьютерные алгоритмы для решения конкретных технических задач $[3,8,14]$. Объектом исследований являются конструкции гидромелиоративных системы с расширенным функционалом по регулированию гидротермического режима агрофитоценоза. Предмет исследований - компьютерные алгоритмы совершенствования конструкций технических систем для регулирования гидротермического режима агрофитоценоза на основе метода морфологической комбинаторики.

Метод морфологической комбинаторики предполагает выделение совокупностей конструктивных или каких-либо других признаков технической системы, причем для каждого из признаков подбираются альтернативные варианты. Для решения задачи в автоматическом режиме важно, чтобы можно было организовать перебор вариантов исполнения этих признаков. То есть необходимо, чтобы варианты реализации конструктивного признака характеризовались каким-либо количественным шагом, либо чтобы эти варианты были организованы в списки, которые были бы доступны перебору.

Конструктивные признаки группируются относительно функциональных элементов технической системы. Чтобы определить конструктивные признаки, используют некоторые общие подходы. В частности, оценивается взаимное расположение функциональных элементов, составляющих техническую систему. Качественная либо 
количественная характеристика взаимного расположения функциональных элементов системы является конструктивным признаком. Если техническую систему можно разбить на функциональные элементы, то, безусловно, будут и взаимосвязи между ними. То, как устроены эти связи, тоже является конструктивным признаком технической системы. К конструктивным признакам относится геометрическая форма, соотношение размерных оценок, материал, из которого изготовлен конструктивный элемент. Эти общепринятые сегменты не ограничивают поиска конструктивных признаков, причем для каждой технической системы всегда можно сформировать свою систему наиболее важных параметров.

Результаты и обсуждение. Общая последовательность формирования И-ИЛИдерева, являющегося основным инструментом морфологического синтеза технических решений, может быть использована при разработке новых конструкций гидромелиоративных систем для регулирования гидротермического режима агрофитоценоза (рисунок 1). В основу этой работы положена функциональная модель технической системы. Такая модель относительно технической системы для регулирования гидротермического режима агрофитоценоза была разработана нами в рамках работы над созданием концепта одноименной системы, а основные результаты этих исследований опубликованы ранее [2]. Функциональная модель, анализ технологических функций гидромелиоративной системы для регулирования гидротермического режима агрофитоценоза позволяют выделить основные функциональные элементы такого рода конструкций для их дальнейшего совершенствования и поиска новых технических решений.

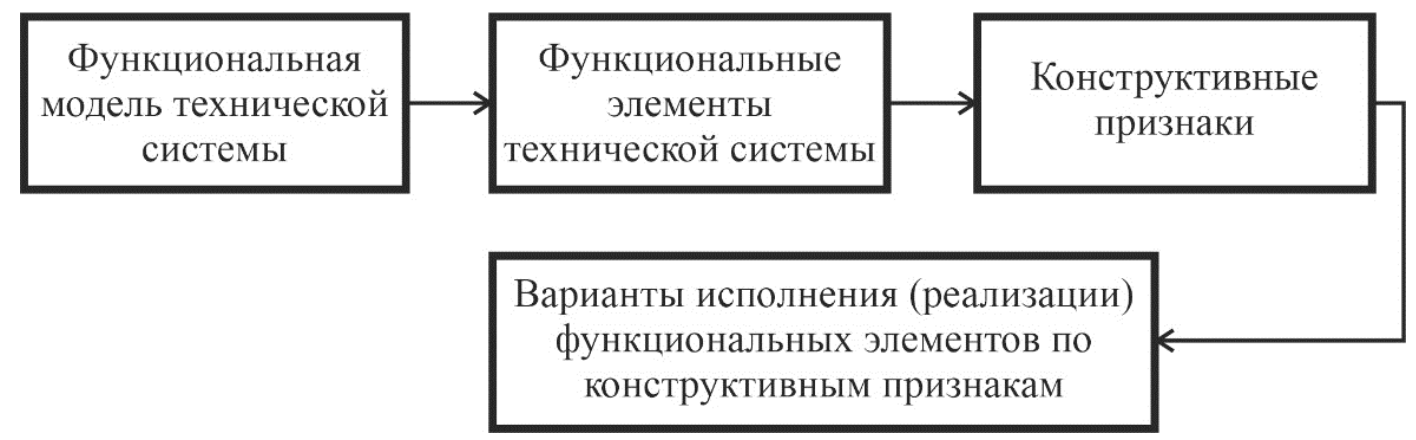

Рисунок 1 - Укрупненная модель построения И-ИЛИ-дерева морфологического синтеза технических решений для регулирования гидротермического режима агрофитоценоза

Figure 1 - An enlarged model for constructing an AND-OR-tree of morphological synthesis of technical solutions for regulating the hydrothermal regime of agrophytocenosis

Конструкции технической системы для регулирования гидротермического режима агрофитоценоза рассматриваются в качестве составляющих исполнительных сегментов гидромелиоративных систем нового поколения. В самом общем случае этот сегмент может быть как «веткой» гидромелиоративной системы нового поколения, так и представлять всю систему в целом, в зависимости от масштабирования новых технологий в практику орошаемого земледелия. Конструкции технической системы для регулирования гидротермического режима агрофитоценоза можно с успехом использовать при восстановлении утративших функции сегментов действующих гидромелиоративных систем. Поэтому разрабатываемые технические решения должны быть в первую очередь встраиваемыми в конструкции гидромелиоративных систем нового поколения и лишь во вторую - путем полного поглощения - представлять гидромелиоративную систему в целом. 
Исследованиями выделены следующие функциональные элементы системы для регулирования гидротермического режима агрофитоценоза, техническое развитие которых наиболее значимо в плане повышения эффективности новых технологий:

- водовыпуск системы капельного типа. Концепт технической системы для регулирования гидротермического режима агрофитоценоза предполагает реализацию возможности сочетанного использования сразу нескольких способов полива для достижения наилучших результатов. Реализация сразу нескольких способов полива требует особой организации системы водовыпусков, а также сочетания водовыпусков разного типа. Капельный способ орошения относится к одним из наиболее перспективных гидромелиоративных технологий, предполагающих развертывание на стационарной основе. Это инструмент наиболее гибкого регулирования водного режима почвы, уже сейчас эти технологии высоко автоматизированы и позволяют организовать водообеспечение растений в соответствии с их биологическими потребностями. Использование этого способа полива положено в основу технических систем для комплексного регулирования гидротермического режима агрофитоценоза. При использовании в сочетании с другими способами полива к капельному водовыпуску предъявляются особые требования, и некоторые и них специфичны для решаемой задачи. Поэтому конструктивное совершенствование системы капельных водовыпусков, трансформация технических решений в соответствии с новыми требованиями является важным этапом развития технических систем для регулирования гидротермического режима агрофитоценоза;

- водовыпуск системы дождевального типа. Капельное орошение имеет много преимуществ. В качестве одного из таких преимуществ позиционируется возможность подачи оросительной воды непосредственно в прикорневую зону растений, без распыления влаги в воздухе. Действительно, при таком способе подачи воды смачивается только та зона почвы, в которой расположены корни сельскохозяйственных растений, снижается расход воды на эвапорацию, исключаются потери воды на испарение в воздухе. Однако, такой способ подачи воды имеет и обратную сторону, так как не обеспечивается возможность смачивания растительного покрова, регулирования влажности воздуха и микроклимата в среде посева. Поэтому введение дополнительной системы из водовыпусков дождевального типа является необходимым для создания технологий, направленных на регулирование гидротермического режима агрофитоценоза. При этом реализуется важное преимущество: система дождевальных водовыпусков может быть задействована только при необходимости регулирования микроклимата, компенсации вредного влияния воздушных засух и других неблагоприятных условий среды, тогда как основной полив проводится капельным способом;

- поливной трубопровод является еще одним важным функциональным элементом технической системы для комплексного регулирования гидротермического режима агрофитоценоза. Регулирование таких факторов, как микроклимат посева, ввиду высокой динамики процессов, целесообразно, преимущественно, на площадной основе, когда регуляторы одновременно задействованы на достаточно большой территории. Использование стационарных систем орошения ввиду этого является приоритетным для регулирования гидротермического режима региона, а поливной трубопровод - неотъемлемым функциональным элементом таких технических систем. В отличие от других систем орошения, системы для комплексного регулирования гидротермического режима агрофитоценоза предполагают устройство на поливных трубопроводах сразу нескольких типов водовыпусков. Требования к таким трубопроводам иные в сравнении с какими-либо до этого использовавшимися. Поэтому конструктивное совершенствование системы поливных трубопроводов видится одним из перспективных направлений 
технического развития конструкций, обеспечивающих расширение функционала современных гидромелиоративных систем в плане регулирования гидротермического режима агрофитоценоза;

- распределительный трубопровод. Это важный функциональный элемент системы, так как отвечает за распределения водотока в пределах поливного модуля. Организация этого функционального элемента тесно связана с конструктивными решениями функциональных элементов системы в самом поливном модуле. На уровне этого элемента может осуществляться регулирование дифференцированных по напору потоков и от конструкции его исполнения во многом зависит эффективность системы в целом;

- система добавочного водообеспечения. Это функциональный элемент, специфика которого определяется особенностями компоновки гидромелиоративной системы для регулирования гидротермического режима агрофитоценоза. Сама потребность в этой функции специфична для систем, обеспечивающих комплексное регулирование гидротермического режима, и связана с увеличением расхода воды при одновременной работе водовыпусков капельного и дождевального типа. В зависимости от режима работы система должна обеспечивать разную производительность по пропуску объемов оросительной воды. Кроме того, предполагается, что система добавочного водообеспечения сможет обеспечить потребности в оросительной воде при кратковременных включениях дождевателей при регулировании микроклимата в среде посева на уровне поливного модуля без необходимости создания напоров в водопроводящих трубопроводах;

- система дифференцированных напоров. Этот функциональный элемент технической системы также является специфичным для конструкций, обеспечивающих комплексное регулирование гидротермического режима агрофитоценоза. Использование разных способов распределения воды по орошаемому участку и, более того, разные главные функции самого орошения определяет потребность и в разных энергетических составляющих распределяемого водного потока. В этом плане система должна обеспечивать возможность дифференцирования напорно-расходных характеристик в соответствии с заданными режимами орошения;

- система фильтрации воды. Это чрезвычайно важный функциональный элемент для систем капельного орошения и мелкодисперсного дождевания, имеющих малое проходное сечение водовыпусков. Отсутствие механических и, что не менее важно, органических примесей является необходимым условием надежной работы гидромелиоративной системы, обеспечивающей реализацию расширенного функционала по регулированию гидротермического режима агрофитоценоза. Важно также учитывать и расход энергии, затрачиваемой при прохождении воды через систему очистки, что отражается на изменении расходно-напорных характеристик водного потока. Выбор оптимальной конструкции системы водоочистки в этом плане видится весьма перспективным направлением развития технических систем для комплексного регулирования гидротермического режима агрофитоценоза;

- гидроподкормщик. Гидромелиоративные системы с расширенным функционалом, обеспечивающим регулирование гидротермического режима агрофитоценоза, как уже говорилось, предполагают комбинирование различных способов орошения. Рассматриваются варианты с дозированным (капельным) распределением воды непосредственно в прикорневую зону почвы, с распределением воды в форме капель дождя, создание мелкокапельной взвеси, осаждающейся на вегетативных органах растений. Все эти способы обеспечивают уникальную возможность проведения подкормок питательными элементами в рамках как корневых, так и некорневых технологий. Безусловно, различные технологии подкормок должны быть ориентированы на использование раз- 
личных комплексов питательных макро- и микроэлементов. В этом смысле конструкция гидроподкормщика, варианты его интеграции с мелиоративной системой должны наилучшим образом обеспечить возможность комплексного использования различных технологий подкормки сельскохозяйственных растений;

- водоводы проводящей оросительной сети разных порядков. Как правило, это наиболее материало- и энергоемкий функциональный элемент практически любой гидромелиоративной системы. От конструкции этого функционального элемента зависят объемы непроизводительных потерь воды, энергетический КПД водотока, экология окружающей среды и другие важные факторы, определяющие эффективность гидромелиоративных технологий. Гидромелиоративные системы с расширенным функционалом, обеспечивающие регулирование гидротермического режима агрофитоценоза, предъявляют особые требования к водопроводящей сети в плане сохранения расходнонапорных характеристик при транспортировании оросительной воды. Следует учитывать и необходимость изменения напорно-расходных характеристик водотока при разных режимах работы системы, а значит и транспортная функция при этом должна выполняться, как минимум с близкими значениями КПД.

Для каждого из функциональных элементов системы предполагается выделение набора конструктивных признаков с различными вариантами реализации. В первую очередь выделяются важные с точки зрения выполнения функции конструктивные признаки. Однако, ранжирование по «важности» является исключительно субъективным фактором, в силу чего такой подход не всегда оправдан. Метод морфологической комбинаторики предполагает необходимость составления наиболее полного перечня конструктивных признаков, хотя «полнота» этого перечня также является признаком субъективным, в значительной мере зависящим от квалификации составителя. В этом смысле наилучшим выходом является организация коллективной работы над формированием перечня конструктивных признаков, а значит, информационная система должна:

- иметь возможность расширения перечня конструктивных признаков;

- обеспечить доступ и согласованную работу экспертного коллектива.

Для каждого конструктивного признака должен быть сформирован перечень вариантов реализации. Если конструктивный признак в настоящем имеет только один вариант реализации, то он не используется при составлении возможных комбинаций в конструкции технической системы. Списки вариантов реализации конструктивного признака также должны быть расширяемыми, что не исключает потенциальную возможность добавления реализаций и включения конструктивного признака в работу при составлении комбинаций конструкции. В плане вариантов реализации конструктивных признаков необходимо отметить еще один момент. Дело в том, что можно использовать реальные (действительно исполнимые в настоящем) варианты реализации, а можно идеализированные. Например, варианты реализации по конструкционным материалам функционального элемента могут включать только известные материалы, а может быть задан неизвестный в настоящем конструкционный материал с «придуманными», идеальными характеристиками. Второй подход существенно расширяет возможности технического творчества и, на наш взгляд, предпочтительнее в научных исследованиях. При этом оцениваются конкурентный потенциал несуществующего «варианта реализации» конструктивного признака и составляются исходные требования на его создание.

Варианты реализации конструктивных признаков по всем выделенным функциональным элементам в различных комбинациях составляют вариации конструктивного исполнения создаваемой технической системы. Алгоритм синтеза и идентификации вариантов реализации технической системы для регулирования гидротермического ре- 
жима агрофитоценоза приведен на рисунке 2. В основе алгоритма также лежит функциональная модель технической системы, которая позволяет выделить основные функциональные элементы. Алгоритм работает со списками, составленными в древовидные структуры данных с иерархической организацией. Функциональные элементы технической системы составляют первый (высший) уровень организации данных. Ветвление древовидной структуры данных на этом уровне касательно технической системы для комплексного регулирования гидротермического режима агрофитоценоза соответствует числу вышеперечисленных функциональных элементов.

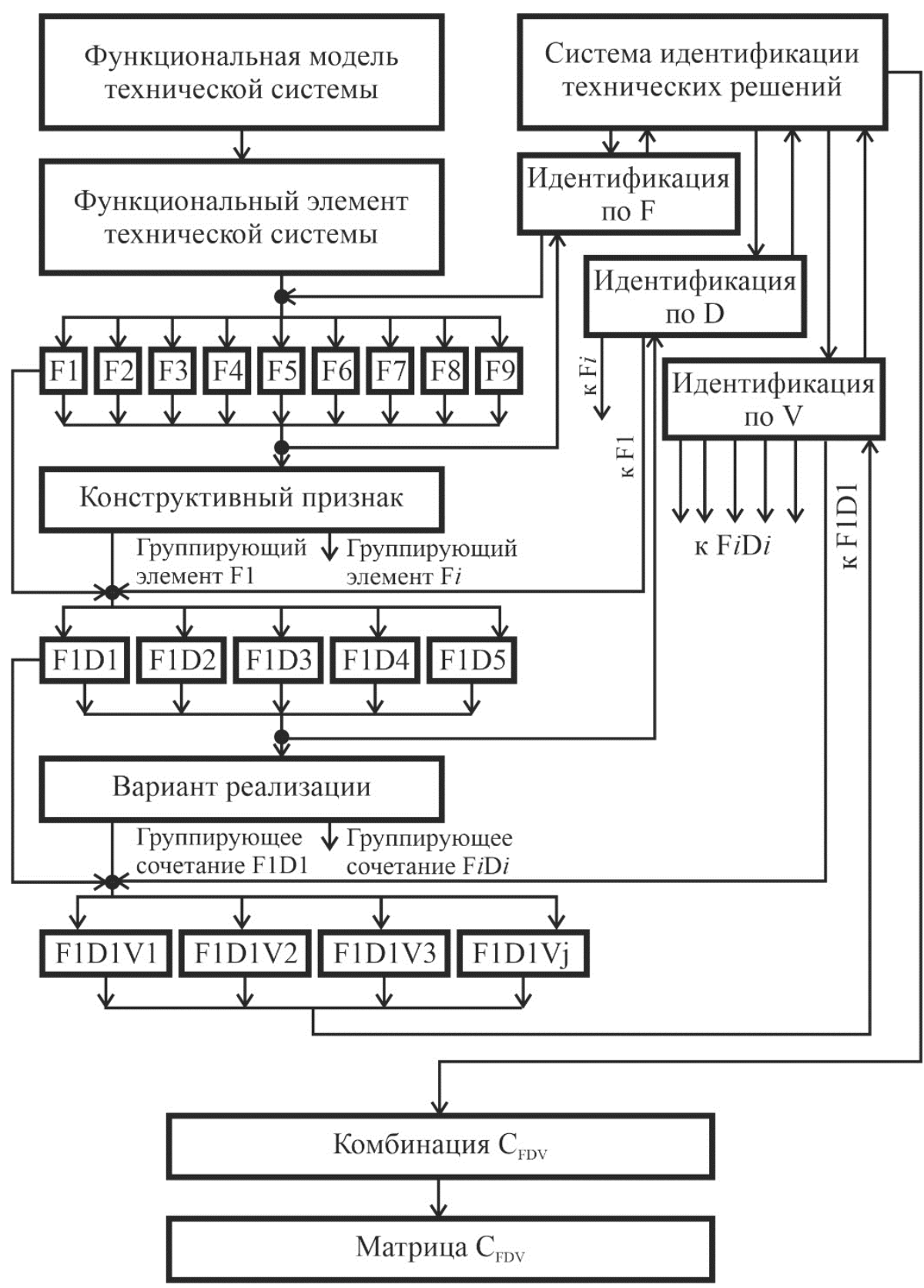

Рисунок 2 - Алгоритм синтеза и идентификации вариантов реализации технической системы для регулирования гидротермического режима агрофитоценоза

Figure 2 - Algorithm for the synthesis and identification of options for the implementation of a technical system for regulating the hydrothermal regime of agrophytocenosis 


\section{***** НЗВЕСТИЯ ***** \\ НИЖНЕВОАЖСКОГО АГРОУНИВЕРСИТЕТСКОГО КОМПАЕКСА: НАУКА И ВЫСШЕЕ ПРОФЕССИОНААЬНОЕ ОБРАЗОВАНИЕ}

Параллельно древовидной организации данных алгоритм реализует систему идентификации технических решений, построенную на комбинации трех кодов. Идентификация технических решений по коду $\mathrm{F}$ определяет как раз функциональный элемент системы, относительно которого организуется перебор вариантов реализации конструктивных признаков. Каждому функциональному элементу присваивается номер-идентификатор с кодом идентификатора F. Структурированные таким образом данные с присвоенным каждой структуре кодом-идентификатором являются группирующим элементом для образования структур данных следующего порядка. Это, так называемое, вторичное ветвление древовидной структуры данных, упорядочивающее информацию по перечню конструктивных признаков для каждого из функциональных элементов технической системы. Идентификатором для этой группы данных является код D.

Каждый элемент с кодом идентификатора F может иметь совокупность информационных структур с кодом идентификатора D. Каждой информационной структуре с кодом идентификатора D присваивается индивидуальный номер-идентификатор. Алгоритм присвоения такого номера может предполагать какое-то ранжирование данных, например, по важности, перспективности и т.д. Однако последнее необязательно, и номер идентификатора может присваиваться в абсолютно случайном порядке. Поскольку список конструктивных признаков для каждого функционального элемента системы является расширяемым, то и ограничений в номерах идентификатора с кодом D алгоритм не предполагает.

Сочетание идентификаторов $\mathrm{F}$ и $\mathrm{D}$ в свою очередь является группирующим комплексом для структур данных следующего уровня, которому условно присвоен код $\mathrm{V}$. Данные этого уровня являются вариантами реализации конструктивных признаков для каждого из функциональных элементов системы. При этом функциональный элемент системы задается кодирующим идентификатором $\mathrm{F}$, а конструктивный признак функционального элемента, кодирующим идентификатором D. Структуры данных, характеризующих варианты реализации конструктивных признаков, также имеют расширяемый формат и могут дополняться в процессе совершенствования технической системы. В связи с этим даже найденный на каком-то этапе оптимальный вариант технической системы, не может быть признан наилучшим решением, так как, возможно, с расширением списков будет создана новая конструкция, лучше отвечающая поставленным задачам.

Синтезированная по описанному алгоритму комбинация CFDV характеризует новое техническое решение с реализацией технической инновации в функциональном элементе системы F. Представленный алгоритм позволяет не только синтезировать, но и идентифицировать всю возможною совокупность технических решений, определяемых всеми возможными сочетаниями «реализаций» конструктивных признаков по каждому функциональному элементу. Итогом решения представленного алгоритма является матрица комбинаций CFDV технической системы для комплексного регулирования гидротермического режима агрофитоценоза. Матрица CFDV является основой алгоритма создания технических систем для регулирования гидротермического режима агрофитоценоза на основе метода морфологической комбинаторики (рисунок 3 ).

Алгоритм предполагает организацию перебора всех комбинаций возможных реализаций конструктивных признаков функциональных элементов технической системы для регулирования гидротермического режима агрофитоценоза. Нулевой шаг алгоритма заключается в присвоении кодам-идентификаторам F, D и V номеров, равным 1; соответственно формируется начальная комбинация F1D1V1. Эта комбинация принимается в качестве «точки отсчета» при организации перебора всех сочетаний в рамках, 
доступных на момент расчета списков по V (вариант реализации конструктивного признака) и по D (конструктивный признак функционального элемента). Одновременно осуществляется запуск и по второй ветке алгоритма, реализующей генерацию начального технического решения.

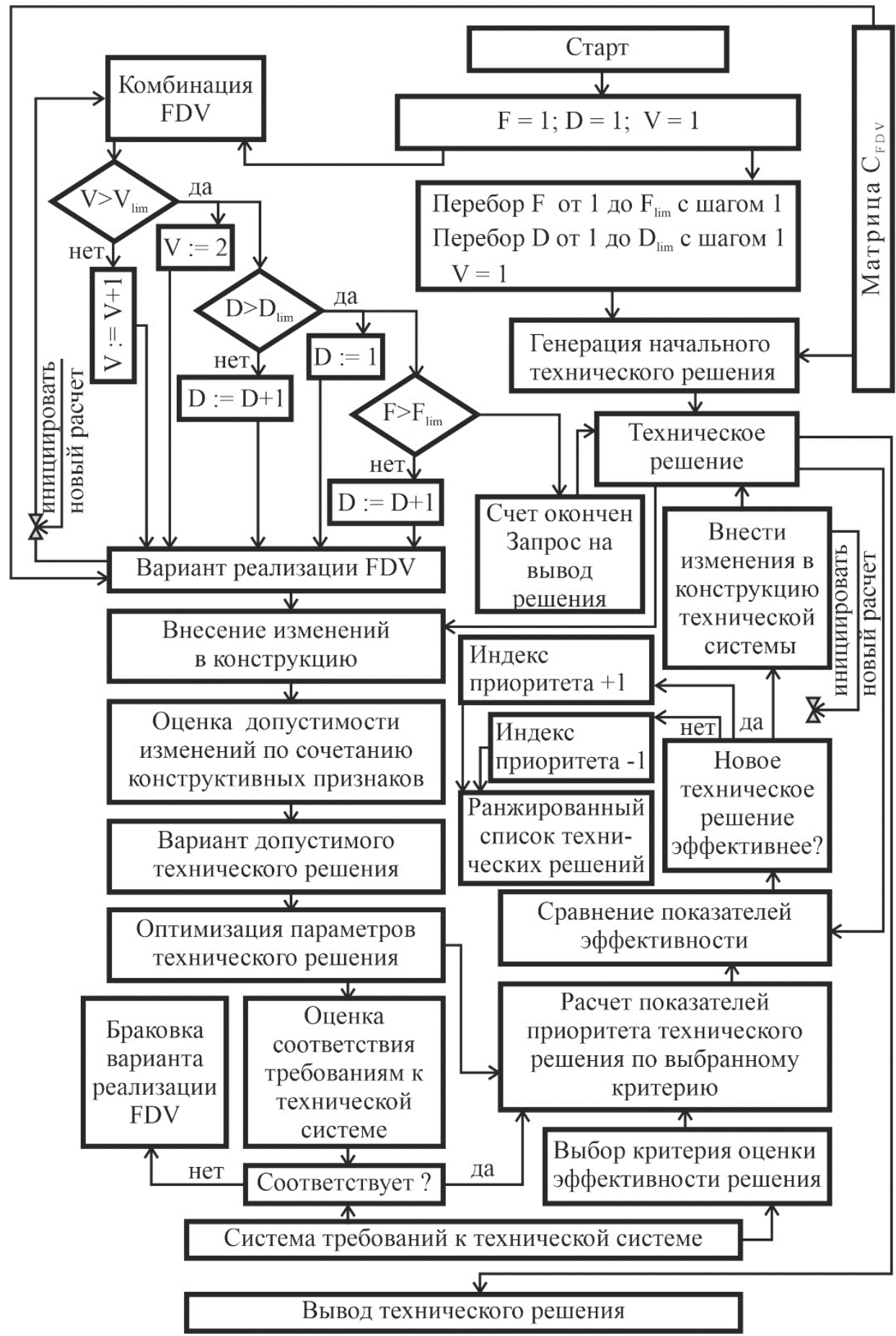

Рисунок 3 - Алгоритм создания технических систем для регулирования гидротермического режима агрофитоценоза на основе метода морфологической комбинаторики

Figure 3 - Algorithm for creating technical systems for regulating the hydrothermal regime of agrophytocenosis based on the method of morphological combinatorics 
Для генерации начального технического решения алгоритм осуществляет перебор всех функциональных элементов системы (F) и, соответствующих им, конструктивных признаков (перебор по D). При этом для каждого сочетания F и D варианту реализации конструктивного признака (V) присваивается номер, равный 1. Целесообразно в качестве варианта реализации конструктивного признака с номером 1 принять вариант известного технического решения. Тогда это, уже проверенное техническое решение, может использоваться в качестве контроля для сравнения с техническими решениями, имеющими те или иные инновационные реализации. Генерация технического решения предполагает использование данных матрицы CFDV, которые, помимо кода идентификатора, содержат исчерпывающее описание используемых реализаций конструктивных признаков для каждого функционального элемента технической системы. Сгенерированное техническое решение передается в одноименный блок «Техническое решение», который является центральным звеном всего алгоритма. В результате, во временную память блока «Техническое решение» записывается код и исчерпывающее инженерное описание начального технического решения.

Перебор комбинаций FDV в первой из рассматриваемых веток алгоритма организован стандартным образом. В первую очередь осуществляется перебор по списку реализаций конструктивного признака функционального элемента технической системы (перебор по V). При этом если значение кода V достигает предельного значения Vlim и выходит за пределы существующего списка, алгоритм переходит к следующему конструктивному признаку $(\mathrm{D}+1)$. При этом коду $\mathrm{V}$ (вариант реализации конструктивного признака) присваивается значение 2. Значения V1 игнорируются, так как их комбинация составляет начальное решение технической системы. При достижении предела перебора списка по $\mathrm{D}$ ( $\mathrm{D} \geq \mathrm{Dlim})$, то есть список выделенных конструктивных признаков функционального элемента исчерпан, алгоритм переходит к следующему функциональному элементу технической системы $(\mathrm{F}+1)$.

При этом коду D присваивается значение 1 , а коду V - значение 2. Перебор по списку конструктивных признаков функционального элемента системы и вариантам их реализации повторяется. При исчерпании списка выделенных функциональных элементов системы счет алгоритма считается оконченным и инициируется запрос на вывод наилучшего технического решения.

За один цикл алгоритм перебора формирует одну новую комбинацию FDV. Эта комбинация определяет функциональный элемент и конструктивный признак, для которого выбирается новый вариант реализации. Использование нового варианта реализации конструктивного признака и является элементом инновации, отличающим новое техническое решение от предыдущего. Для данного варианта из матрицы CFDV импортируются данные, включающие техническое описание варианта реализации конструктивного признака, что позволяет перейти к следующему шагу выполнения алгоритма - внесению изменений в конструкцию технической системы. На этом этапе импортируются данные по текущему техническому решению и в него вносятся изменения, соответствующие новой реализации конструктивного признака. По сути формируется сырой вариант нового технического решения.

На следующем этапе алгоритм предполагает проверку по поводу допустимости внесения в конструкцию изменений, соответствующих новой реализации конструктивного признака. Вероятно, эта реализация не возможна ввиду особенностей конструкции других функциональных модулей, или не сочетается с текущей версией реализации других конструктивных признаков функционального модуля, в который вносятся изменения. Если внесение изменение допустимо, алгоритм переходит к оптимизации пара- 
метров технического решения. Действительно, внесение конструктивных инноваций предполагает возможность изменения режимов работы функционального модуля. И этот новый вариант технического решения уже нельзя считать оптимизированным по совокупности конструктивных параметров, учитывающих новый вариант реализации конструктивного признака. Алгоритм оптимизации конструктивных параметров системы был разработан и опубликован нами ранее; его логика и возможности вполне отвечают поставленным здесь задачам [1].

Оптимизированное, с точки зрения режимных параметров, техническое решение проходит проверку на соответствие требованиям, предъявляемым к техническим системам с расширенным функционалом по регулированию гидротермического режима агрофитоценоза. Если полученное в результате комбинаторного замещения вариантов реализации конструктивных признаков техническое решение не соответствует системе предъявляемых требований, то вариант бракуется.

Если полученное в результате внесения изменений техническое решение соответствует системе предъявляемых требований, то алгоритм осуществляет переход к следующему этапу расчетов. На следующем этапе осуществляется расчет показателей приоритета технического решения. Критерий приоритета в зависимости от решаемых задач может быть разный, но формируется он с учетом требований к технической системе для регулирования гидротермического режима агрофитоценоза. Критерий приоритета может выбираться на основании главного фактора, а также может быть комплексирован из совокупности факторов. Величина критерия приоритета определяется для технического решения с уже оптимизированными конструктивными параметрами. Это позволяет использовать расчетную величину критерия приоритета для сравнения предшествующего и нового технического решения с внесенными в конструкцию изменениями, соответствующими текущей комбинации FVD. В случае если величина критерия приоритета нового технического решения окажется ниже, чем у предшествующего, то такой вариант реализации системы отвергается. Собственно, само новое решение в этом случае занимает соответствующее своему рангу место в ранжированном списке реализаций конструкции.

Если новое техническое решение окажется более приоритетным, чем предшествующее, то принимается решение о внесении изменений в конструкцию технической системы. При этом данные нового технического решения замещают всю информацию по предшествующему техническому решению в блоке «Техническое решение». Одновременно инициируется запрос на новый цикл расчета с перебором комбинаций FDV, и все действия в последовательности алгоритма повторяются.

Как уже говорилось, блок «Техническое решение» - это один из центральных блоков настоящего алгоритма. Последовательное замещение данных по техническим решениям на более эффективные, от цикла к циклу, позволяет в итоге выявить наилучшее техническое решение, которое и станет итогом решения всего алгоритма. Расчет считается законченным при переборе всех вариантов реализации конструктивных признаков по всем спискам конструктивных признаков для каждого из выделенных функциональных элементов технической системы. По завершении всех циклов расчета дается команда на вывод наилучшего технического решения. Следует добавить, что расширение списков возможных реализаций конструктивных признаков, как и списка самих признаков, может существенно повлиять на результаты работы алгоритма. Могут быть получены новые, более эффективные технические решения. В этом смысле предложенный алгоритм позволяет автоматизировать работу с наиболее трудоемкими, рутинными расчетами, но ни в коем случае не подменяет процесс инженерного творчества. 
Выводы. Таким образом, известный в инженерии и хорошо апробированный метод морфологической комбинаторики является перспективным инструментом при проектировании технических систем для регулирования гидротермического режима агрофитоценоза. Такие конструкции разрабатываются как составляющие гидромелиоративных систем нового поколения и, в общем, ориентированы как на восстановление утративших функции сегментов действующих систем орошения, так и на создание гидромелиоративной системы в целом. В основу создания гидромелиоративных систем, обеспечивающих комплексное регулирование гидротермического режима агрофитоценоза, положен принцип комбинирования различных способов орошения. Такие системы сложны в конструктивном исполнении и предъявляют особые требования к работе всех функциональных элементов, имеют принципиально новые функциональные блоки в сравнении с известными конструкциями гидромелиоративных систем. Как и в любой сложной технической системе, создаваемые технические решения имеют множество альтернативных вариантов реализаций, причем изменения даже в самом незаметном конструктивном модуле функционального элемента могут существенно отражаться на работе всей системы в целом. Исследованиями разработан алгоритм создания технических систем для регулирования гидротермического режима агрофитоценоза на основе метода морфологической комбинаторики. Алгоритм включает систему идентификации вариантов реализации технической системы для регулирования гидротермического режима агрофитоценоза, функциональные модули перебора комбинаций возможных конструктивных реализаций, оценки согласованности конструктивных решений в различных комбинациях возможных реализаций, модуля оптимизации конструктивных параметров при введении изменений по оцениваемому варианту реализации, модуля оценки соответствия требованиям, предъявляемым к таким техническим системам, модулей оценки критерия приоритета технического решения, а также сравнения нового и предшествующего технического решения по этому критерию. Алгоритм построен так, что техническое решение, отличающееся большим приоритетом, всегда замещает техническое решение с меньшим приоритетом. В итоге это позволяет найти наилучшее техническое решение при всех возможных вариантах реализации конструктивных признаков для совокупности функциональных элементов системы. Алгоритм позволяет автоматизировать процесс поиска наилучших конструктивных решений при создании гидромелиоративных систем для комплексного регулирования гидротермического режима агрофитоценоза.

\section{Библиографический список}

1. Бородычев В. В., Лытов М. Н. К решению задачи автоматизации вычислений при проектировании систем комбинированного орошения // Известия Нижневолжского агроуниверситетского комплекса: Наука и высшее профессиональное образование. 2020. № 4 (60). С. 291-304.

2. Бородычев В. В., Лытов М. Н. Технологические функции технической системы для регулирования гидротермического режима агрофитоценоза и комплексной протекции посевов от климатических рисков // Известия Нижневолжского агроуниверситетского комплекса: Наука и высшее профессиональное образование. 2020. № 2 (58). С. 307-319.

3. Галочкин В. И. Перечисление решающих деревьев на И-ИЛИ дереве по монотонным ограничениям // Программные системы и вычислительные методы. 2016. № 4. С. 340-347.

4. Куртебаев Б. М. Испытания модульных систем мелкодисперсного дождевания // Известия ВУЗов Кыргызстана. 2017. № 7. С. 84-86.

5. Майер А. В., Захаров Ю. И., Криволуцкая Н. В. Универсальная многофункциональная система орошения для комбинированных способов полива // Известия Нижневолжского агроуниверситетского комплекса: Наука и высшее профессиональное образование. 2015. № 1 (37). C. 206-210. 
6. Макарычев С. В. Особенности комбинированного механизма тепловлагообмена в почве при использовании гидромелиораций // Вестник Алтайского государственного аграрного университета. 2019. № 11 (181). С. 62-67.

7. Муродов Н. К., Авлакулов М. Анализ теплового режима почвы при орошении сельскохозяйственных культур методом субирригации // Новый университет. Серия: Технические науки. 2016. № 1 (47). С. 12-16.

8. Пальчевский Б. А., Великий А. А. Разработка системы автоматизированного оптимизационного синтеза структуры технических объектов путем использования деревьев "И-ИЛИ" // Технологічні комплекси. 2011. № 2 (4). С. 023-030.

9. Система комбинированного орошения и эффективность производства овощной продукции / Н. Н. Дубенок, А. В. Майер, В. М. Гуренко, С. В. Бородычев // Известия Нижневолжского агроуниверситетского комплекса: Наука и высшее профессиональное образование. 2019. № 2 (54). C. 253-265.

10. Терпигорев А. А., Гжибовский С. А., Грушин А. В. Технологии и технические средства для защиты растений в термически напряжённые периоды // Плодоводство и ягодоводство России. 2017. Т. 51. С. 327-332.

11. Учет изменений климата и интенсивности засух при планировании орошения в зоне южной степи Украины / О. И. Жовтоног, Л. А. Филиппенко, Т. Ф. Деменкова, В. В. Полищук, Я. А. Бутенко // Меліорація і водне господарство. 2018. № 1 (107). С. 37-46.

12. Khanal S., Fulton J., Shearer S. An overview of current and potential applications of thermal remote sensing in precision agriculture // Computers and Electronics in Agriculture. 2017. V. 139. P. 22-32.

13. Sahaar S. A., Niemann J. D. Impact of regional characteristics on the estimation of rootzone soil moisture from the evaporative index or evaporative fraction // Agricultural Water Management. 2020. V. 238. N 106225.

14. Shentsova O. M. The heuristic methods in engineering and design as a trend in modern architectural education // Modern Science. 2019. № 1. P. 136-141.

Conclusions. Thus, the well-known in engineering and well-tested method of morphological combinatorics is a promising tool in the design of technical systems for regulating the hydrothermal regime of agrophytocenosis. Such structures are being developed as components of new generation irrigation and drainage systems and, in general, are focused both on the restoration of segments of existing irrigation systems that have lost their functions, and on the creation of an irrigation and drainage system as a whole. The basis for the creation of irrigation and drainage systems that provide comprehensive regulation of the hydrothermal regime of agrophytocenosis is based on the principle of combining various methods of irrigation. Such systems are complex in design and impose special requirements on the operation of all functional elements; they have fundamentally new functional blocks in comparison with the known designs of irrigation and drainage systems. As in any complex technical system, the created technical solutions have many alternative implementations, and changes in even the most imperceptible structural module of a functional element can significantly affect the operation of the entire system as a whole. Research has developed an algorithm for creating technical systems for regulating the hydrothermal regime of agrophytocenosis based on the method of morphological combinatorics. The algorithm includes a system for identifying options for the implementation of a technical system for regulating the hydrothermal regime of agrophytocenosis, functional modules for enumerating combinations of possible design implementations, assessing the consistency of design solutions in various combinations of possible implementations, a module for optimizing design parameters when introducing changes for the evaluated implementation option, a module for assessing compliance with the requirements to such technical systems, modules for evaluating the priority criterion of a technical solution, as well as comparing a new and previous technical solution according to this criterion. The algorithm is constructed in such a way that a technical solution with a higher 
priority always replaces a technical solution with a lower priority. As a result, this allows you to find the best technical solution for all possible options for the implementation of design features for a set of functional elements of the system. The algorithm allows automating the process of finding the best design solutions when creating irrigation and drainage systems for the integrated regulation of the hydrothermal regime of agrophytocenosis.

\section{References}

1. Borodychev V. V., Lytov M. N. K resheniyu zadachi avtomatizacii vychislenij pri proektirovanii sistem kombinirovannogo orosheniya // Izvestiya Nizhnevolzhskogo agrouniversitetskogo kompleksa: Nauka i vysshee professional'noe obrazovanie. 2020. № 4 (60). P. 291-304.

2. Borodychev V. V., Lytov M. N. Tehnologicheskie funkcii tehnicheskoj sistemy dlya regulirovaniya gidrotermicheskogo rezhima agrofitocenoza i kompleksnoj protekcii posevov ot klimaticheskih riskov // Izvestiya Nizhnevolzhskogo agrouniversitetskogo kompleksa: Nauka i vysshee professional'noe obrazovanie. 2020. № 2 (58). P. 307-319.

3. Galochkin V. I. Perechislenie reshayuschih derev'ev na I-ILI dereve po monotonnym ogranicheniyam // Programmnye sistemy i vychislitel'nye metody. 2016. № 4. P. 340-347.

4. Kurtebaev B. M. Ispytaniya modul'nyh sistem melkodispersnogo dozhdevaniya // Izvestiya VUZov Kyrgyzstana. 2017. № 7. P. 84-86.

5. Majer A. V., Zaharov Yu. I., Krivoluckaya N. V. Universal'naya mnogofunkcional'naya sistema orosheniya dlya kombinirovannyh sposobov poliva // Izvestiya Nizhnevolzhskogo agrouniversitetskogo kompleksa: Nauka i vysshee professional'noe obrazovanie. 2015. № 1 (37). P. 206-210.

6. Makarychev S. V. Osobennosti kombinirovannogo mehanizma teplovlagoobmena v pochve pri ispol'zovanii gidromelioracij // Vestnik Altajskogo gosudarstvennogo agrarnogo universiteta. 2019. № 11 (181). P. 62-67.

7. Murodov N. K., Avlakulov M. Analiz teplovogo rezhima pochvy pri oroshenii sel'skohozyajstvennyh kul'tur metodom subirrigacii // Novyj universitet. Seriya: Tehnicheskie nauki. 2016. № 1 (47). P. 12-16.

8. Pal'chevskij B. A., Velikij A. A. Razrabotka sistemy avtomatizirovannogo optimizacionnogo sinteza struktury tehnicheskih ob'ektov putem ispol'zovaniya derev'ev "I-ILI" // Tehnologichni kompleksi. 2011. № 2 (4). P. 023-030.

9. Sistema kombinirovannogo orosheniya i ]ffektivnost' proizvodstva ovoschnoj produkcii / N. N. Dubenok, A. V. Majer, V. M. Gurenko, S. V. Borodychev // Izvestiya Nizhnevolzhskogo agrouniversitetskogo kompleksa: Nauka i vysshee professional'noe obrazovanie. 2019. № 2 (54). P. 253-265.

10. Terpigorev A. A., Gzhibovskij S. A., Grushin A. V. Tehnologii i tehnicheskie sredstva dlya zaschity rastenij v termicheski napryazhjonnye periody // Plodovodstvo i yagodovodstvo Rossii. 2017. T. 51. P. 327-332.

11. Uchet izmenenij klimata $\mathrm{i}$ intensivnosti zasuh pri planirovanii orosheniya $\mathrm{v}$ zone yuzhnoj stepi Ukrainy / O. I. Zhovtonog, L. A. Filippenko, T. F. Demenkova, V. V. Polischuk, Ya. A. Butenko // Melioraciya i vodne gospodarstvo. 2018. № 1 (107). P. 37-46.

12. Khanal S., Fulton J., Shearer S. An overview of current and potential applications of thermal remote sensing in precision agriculture // Computers and Electronics in Agriculture. 2017. V. 139. P. 22-32.

13. Sahaar S. A., Niemann J. D. Impact of regional characteristics on the estimation of rootzone soil moisture from the evaporative index or evaporative fraction // Agricultural Water Management. 2020. V. 238. N 106225.

14. Shentsova O. M. The heuristic methods in engineering and design as a trend in modern architectural education // Modern Science. 2019. № 1. P. 136-141.

\section{Authors Information}

Borodychev Viktor Vladimirovich, Academician of the Russian Academy of Sciences, Director of the Volgograd Branch of the Federal State Budget Scientific Institution All-Russian Scientific Research Institute of Hydrotechnics and Land Reclamation named after A.N. Kostyakova (400002, Volgograd, Timiryazev St., 9), Doctor of Agricultural Sciences, Professor.

ORCID: https://orcid.org/0000-0002-0279-8090. E-mail: vkovniigim@yandex.ru 
Lytov Michail Nikolaevich, Leading Researcher, Volgograd Branch of the Federal State Budget Scientific Institution All-Russian Scientific Research Institute of Hydraulic Engineering and Land Reclamation A.N. Kostyakova (400002, Volgograd, Timiryazev St., 9), Candidate of Agricultural Sciences, ORCID: https://orcid.org/0000-0003-2743-9825 LytovMN@yandex.ru

\title{
Информация об авторах:
}

Бородычев Виктор Владимирович, академик РАН, директор Волгоградского филиала федерального государственного бюджетного научного учреждения Всероссийский научноисследовательский институт гидротехники и мелиорации им. А. Н. Костякова $(400002$, г. Волгоград, ул. Тимирязева, 9), доктор сельскохозяйственных наук, профессор.

ORCID: https://orcid.org/0000-0002-0279-8090. E-mail: vkovniigim@yandex.ru

Лытов Михаил Николаевич, ведущий научный сотрудник Волгоградского филиала федерального государственного бюджетного научного учреждения Всероссийский научно-исследовательский институт гидротехники и мелиорации им. А. Н. Костякова (400002, г. Волгоград, ул. Тимирязева, 9), кандидат сельскохозяйственных наук, ORCID: https://orcid.org/0000-0003-2743-9825 LytovMN@yandex.ru

DOI: $10.32786 / 2071-9485-2021-02-34$

\section{ANALYSIS OF THE EFFICIENCY OF FILTER LOADS FOR DEFERRIZATION OF WATER FROM UNDERGROUND WATER SOURCES FOR DRIP IRRIGATION SYSTEMS}

\author{
A.S. Ovchinnikov ${ }^{1}$, V.S. Bocharnikov ${ }^{1}$, O.V. Bocharnikova ${ }^{1}$, \\ E.V. Pustovalov ${ }^{1}$, M.A. Denisova ${ }^{1}$ \\ ${ }^{1}$ Volgograd State Agrarian University, Volgograd
}

Received 24.12.2020

Submitted 20.05.2021

\begin{abstract}
Introduction. The use of groundwater in land reclamation practice is complicated by the high content of iron impurities. The presence of iron in irrigation water negatively affects the operation of the main and auxiliary equipment of drip irrigation systems, causes corrosion and premature wear of metal pipes of water distribution systems. In addition, prolonged watering with such water negatively affects the biological development of plants. In order to increase the operational reliability of drip irrigation systems, as well as to obtain the planned yields, when using water from underground water sources for irrigation, special attention should be paid to deferrization. In most cases, deferrization is an energy-intensive, capital-intensive method for treating irrigation water. Therefore, the development of the simplest iron removal technology available to a farm is an urgent task. Object. The object of research is the underground waters of the Volgograd region. Materials and methods. Three samples of filtering material took part in the research as a load: quartz sand with a grain size of 1.0-1.5 mm; natural zeolite with a particle size of 1.5-2.0 mm; pyrolusite $\left(\mathrm{MnO}_{2}\right)$ with a fraction of $1.0 \mathrm{~mm}$. For each sample of the filtering material, two variants of the filtration rate were investigated: $\mathrm{V}=5-9 \mathrm{~m} / \mathrm{h} ; \mathrm{V}=10-15 \mathrm{~m} / \mathrm{h}$. Results and conclusions. Filtration through a sand load at $\mathrm{V}=5-9 \mathrm{~m} / \mathrm{h}$, the total iron index ( $\mathrm{Fe}$ general $)$ was reduced to $0.99 \mathrm{mg} / 1$, at $\mathrm{V}=10-15 \mathrm{~m} / \mathrm{h}-$ to $0.44 \mathrm{mg} / 1$, which does not satisfy the conditions of the maximum permissible concentration. The use of zeolite loading made it possible to extract iron impurities to the required indicator $\left(\mathrm{Fe}_{\text {general }}=0.3 \mathrm{mg} / \mathrm{l}\right)$ at a speed of $\mathrm{V}=10-15 \mathrm{~m} / \mathrm{h}$, when at $\mathrm{V}=5-9 \mathrm{~m} / \mathrm{h}$ this indicator decreased only to $0.37 \mathrm{mg} / 1$. The best results were shown by filters with catalytic pyrolusite loading where the efficiency of removing iron from water was $88.2 \%$ at a speed of $\mathrm{V}=5-9 \mathrm{~m} / \mathrm{h}$, and $93.7 \%$ at a speed of $\mathrm{V}=10-15 \mathrm{~m} / \mathrm{h}$. Thus, to obtain the desired result, filtration should be carried out on filters with a zeolite load at a speed of $\mathrm{V}=10-15 \mathrm{~m} / \mathrm{h}$, or through pyrolusite, while there is no need to use higher filtration rates.
\end{abstract}

Key words: groundwater, drip irrigation system, deferrization, quartz sand, natural zeolite, pyrolusite 